\title{
Palliative care ethical guidelines to assist healthcare practitioners in their treatment of palliative care patients
}

\author{
D J McQuoid-Mason, BCom, LLB, LLM, PhD; N Naidoo, LLB \\ Centre for Socio-Legal Studies, Howard College School of Law, University of KwaZulu-Natal, Durban, South Africa
}

Corresponding author: D J McQuoid-Mason (mcquoidm@ukzn.ac.za)

\begin{abstract}
At the request of the Health Professions Council of South Africa (HPCSA), draft ethical guidelines on palliative care (the draft guidelines) have been produced, to assist healthcare practitioners in their treatment of palliative care patients. These have been submitted to the HPCSA, which has circulated them for comment by the boards and other relevant stakeholders. This article provides a wider audience, including healthcare practitioners and patients involved in palliative care, an opportunity to comment on the draft guidelines. Suggestions should be sent to the corresponding author for onward transmission to the HPCSA.
\end{abstract}

S Afr J Bioethics Law 2019;12(1):14-18. DOI:10.7196/SAJBL.2019.v12i1.660

The draft guidelines on palliative care (the draft guidelines) are based on the National Policy Framework and Strategy on Palliative Care developed by the South African (SA) National Department of Health (NDoH). ${ }^{[1]}$ They draw on the guidelines used in Scotland, ${ }^{[2]}$ the USA, ${ }^{[3]}$ the $\mathrm{UK}_{,}{ }^{[4]}$ the Republic of Ireland ${ }^{[5]} \mathrm{Kenya}_{,}{ }^{[6]}$ Australia ${ }^{[7]}$ and Canada ${ }^{[8]}$ The draft guidelines are also based on the World Health Organization (WHO)'s statements on palliative care, ${ }^{[9,10]}$ and locate palliative care within the paradigm of the four biomedical ethical principles, ${ }^{[1]}$ as recommended in the Kenyan guidelines. ${ }^{[6]}$

The draft guidelines cover the following aspects of palliative care: (i) its definition; (ii) when it is used; (iii) why it is used; (iv) its provision; (v) how it is used; (vii) patient autonomy; (viii) beneficence; (ix) nonmaleficence; and $(x)$ justice or fairness.

\section{Definition of palliative care}

Paragraph 1.1 of the draft guidelines quotes the WHO definition of palliative care as 'an approach that improves the quality of life of patients and their families facing the problems associated with lifethreatening illness, through the prevention and relief of suffering by means of early identification and impeccable assessment and treatment of pain and other problems, physical, psychosocial and spiritual! ${ }^{[9]}$

Paragraph 1.2 refers to the WHO's list of the principles behind palliative care: (i) providing relief from pain and other distressing symptoms; (ii) affirming life, and regarding dying as a normal process; (iii) intending neither to hasten nor postpone death; (iv) integrating the psychological and spiritual aspects of patient care; $(v)$ offering a support system to help patients live as actively as possible until death; (vi) offering a support system to help the family cope during the patient's illness and in their own bereavement; (vii) using a team approach to address the needs of patients and their families, including bereavement counselling, if indicated; (viii) enhancing the quality of life, and possibly also positively influencing the course of illness; and (ix) being applied early in the course of illness, in conjunction with other therapies that are intended to prolong life, such as chemotherapy or radiation therapy, and including those investigations needed to better understand and manage distressing clinical complications. ${ }^{[9]}$

Paragraph 1.3 mentions that the NDoH's National Policy Framework and Strategy on Palliative Care ${ }^{[1]}$ recognises the importance of integrating palliative care as an essential component of health service delivery.

\section{When palliative care is used}

Paragraph 2 deals with when palliative care is used. Paragraph 2.1 refers to the Scottish guidelines ${ }^{[2]}$ that state that palliative care is provided to those living with and dying from any advanced, progressive and incurable condition, regardless of age or setting, and from diagnosis to the end of life. A non-exclusive $\mathrm{WHO}^{[1]}$ list of diseases in which palliative care is used is provided in paragraph 2.2, which refers to: (i) cardiovascular disease; (ii) cancer; (iii) chronic respiratory diseases; (iv) HIV/AIDS; ( $v$ ) diabetes; (vi) kidney failure; (vii) chronic liver disease; (viii) multiple sclerosis; (ix) Parkinson's disease; $(x)$ rheumatoid arthritis; (xi) neurological disease; (xii) dementia; (xiii) congenital anomalies; and (xiv) drug-resistant tuberculosis (TB).

\section{Why palliative care is used}

Paragraph 3.1 explains why palliative care is used, and that palliative care is recognised by the United Nations as part of the human right to health mentioned in the International Covenant on Economic, Social and Cultural Rights (article 12). ${ }^{[12]}$ The goal of palliative care (paragraph 3.2) is to achieve the best possible quality of life for patients and their families, even if life expectancy is short. ${ }^{[1]}$ Paragraph 3.3 is an extension of the right to human dignity (section 10) and access to healthcare (section 27(1)(a)) in the SA Constitution. ${ }^{[13]}$ Paragraph 3.4 observes that the Constitution also guarantees the right to basic healthcare for children (section 28 (1)(c)), including the provision of paediatric palliative care, and not merely access to such care, as is the case with adults. Paragraph 3.5 refers to the observation by the NDoH's National Policy Framework and Strategy on Palliative 
Care $^{[1]}$ that palliative care can reduce unnecessary hospitalisation and use of healthcare services, which lessens the costs to the health system.

\section{Who provides palliative care?}

Paragraph 4.1 bases some observations on who should provide palliative care on the Irish palliative care guidelines, ${ }^{[5]}$ and states that palliative care is provided by all healthcare and social work professionals working with people with life-threatening conditions. From the National Health Policy Framework and Strategy ${ }^{[3]}$ and the Kenyan guidelines, ${ }^{[6]}$ paragraph 4.2 also states that healthcare practitioners providing palliative care should possess the requisite knowledge, skills and attitudes to meet the physical, psychological, practical, social and spiritual needs of their patients, and should keep their skills up to date.

Paragraph 4.4 states that it is because of the inadequate provision of palliative care services in the country that the NDoH has developed a strategy to increase the scope of such services. ${ }^{[1]}$ Paragraph 4.5 echoes the Canadian guidelines, ${ }^{[8]}$ which state that it is imperative that all healthcare professionals play an advocacy role on the importance of palliative care services in the public health sector, and the observations by the Australian position statement ${ }^{[7]}$ and SA National Policy Framework and Strategy guidelines ${ }^{[1]}$ that palliative care should be included in the curriculum of healthcare practitioner training.

\section{Where palliative care is used}

Paragraph 5.1 describes settings where palliative care may be used, and states that it can be provided in any public or private setting, such as hospitals, hospices, a GP's rooms or the patient's home (paragraph 5.2). Paragraph 5.3 refers to the draft NDoH National Policy Framework and Strategy on Palliative Care ${ }^{[1]}$ list of the different palliative care service delivery settings: (i) home based; (ii) mobile outreach services; (iii) outpatient care; (iv) inpatient facilities; ( $v$ ) hospital-based palliative care teams; (vi) day care; (vii) frail care and other care homes; (viii) workplace programmes; and (ix) correctional services.

\section{How palliative care is used}

Paragraph 6.1 states that when healthcare practitioners provide palliative care, they should apply the biomedical ethical principles ${ }^{[11]}$ of autonomy, beneficence, non-maleficence and justice, as in the Kenyan guidelines, ${ }^{[6]}$ and it requires healthcare practitioners to respect the worth, dignity and human rights of their patients. Patients facing life-threatening illnesses, and their families, are likely to be vulnerable and anxious during this time, and healthcare practitioners providing palliative care will find the bioethical principles helpful in guiding decision-making (paragraph 6.2).

Paragraph 6.3 states that healthcare practitioners must deliver care in a manner that upholds these principles, and the rights and values of the patient and their family, even in the midst of a dehumanising hospital environment in urban areas, which may involve high technology, buzzing alarms and complex, end-oflife decision-making, as described by Templeman. ${ }^{[14]}$ Healthcare practitioners must establish trust with the patient and their family through availability, listening, providing honest answers and having a non-judgemental attitude (paragraph 6.4). Healthcare practitioners should also (paragraph 6.5) adhere to the Health Professions Council of SA (HPCSA)'s general ethical guidelines and the NDoH's National Policy Framework and Strategy on Palliative Care. ${ }^{[1]}$

\section{Patient autonomy and palliative care}

Paragraph 7 deals with patient autonomy, as defined by the World Medical Association (WMA). ${ }^{[16]}$ The WMA reiterates that patient autonomy recognises the ability of patients to decide for themselves ${ }^{[16]}$ regarding palliative care, and is in accordance with the constitutional rights ${ }^{[13]}$ to human dignity (section 10) and bodily and psychological integrity (section 12(2), paragraph 7.1.1). Patient autonomy ${ }^{[11]}$ presumes that patients have the required information and capacity to make rational decisions about palliative care, and that the circumstances allow them to give informed consent (paragraph 7.1.2). The draft guidelines (paragraph 7.1.3) remind practitioners that when obtaining informed consent from patients, healthcare practitioners providing palliative care must comply with the National Health Act No. 61 of $2003^{[15]}$ and the HPCSA's guidelines on informed consent. ${ }^{[17]}$

\section{Capacity to consent to palliative care}

Paragraph 7.2 explains the importance of recognising the capacity of patients to consent to palliative care. Healthcare practitioners providing palliative care should assume that every mentally competent adult patient and every sufficiently mature child patient over the age of 12 years has legal capacity to consent to, or oppose, medical interventions - unless it is shown that they clearly cannot understand the information given to them (paragraph 7.2.1). ${ }^{[15]}$ The patient, and not the healthcare practitioner providing palliative care, should determine what is in the patient's best interests (paragraph 7.2.2). Although healthcare practitioners may recommend a course of treatment, they may not pressurise or manipulate the patient into accepting their advice (paragraph 7.2.3). Healthcare practitioners providing palliative care must understand and respect the fact that a patient's decision-making may be guided by values, customs and beliefs different from the practitioner's (paragraph 7.2.4). ${ }^{[16]}$

If a mentally and legally competent patient, who is fully informed of the benefits and risks of treatment, consents to or refuses a particular course of treatment, ${ }^{[15]}$ their decision must be respected, even if the healthcare practitioner providing palliative care believes it will result in serious harm or even death (paragraph 7.2.5). ${ }^{[16]}$ Respecting patient autonomy, however, does not mean that patients are entitled to illegal, unethical or medically inappropriate treatment simply because they have requested it (paragraph 7.2.6). ${ }^{[3]}$ Healthcare practitioners should only recommend and provide treatment options that are scientifically grounded, and are reasonably expected to yield the intended benefits (paragraph 7.2.6). ${ }^{[17]}$

Paragraph 7.2.7 provides that should patients request illegal, unethical or medically inappropriate treatment, the healthcare practitioner providing palliative care should, as in the US guidelines, ${ }^{[3]}$ reassure them and their family of the sound treatment options available - subject to consent by the patient or their surrogate.

\section{Advanced palliative care planning}

Paragraph 7.3.1 emphasises the importance of advanced palliative care planning, and that patient autonomy includes the need to protect patients with diminished autonomy. As in the US guidelines ${ }^{[3]}$ and the HPCSA National Patients' Rights Charter, ${ }^{[18]}$ healthcare practitioners providing palliative care should encourage competent 
patients to indicate their wishes regarding treatment options in an advance directive, ${ }^{[16]}$ such as a living will. Alternatively, they can, in writing, nominate a surrogate in terms of the National Health Act of $2003^{[15]}$ to mandate such a person to make decisions on their behalf if they become incapacitated (paragraph 7.3.2). An advance directive helps to ensure consideration of the patient's values and preferences, even when they lack the capacity to express them (paragraph 7.3.3).

Paragraph 7.3.4 follows the US guidelines, ${ }^{[3]}$ which state that healthcare practitioners providing palliative care should, in their medical records, record conversations with a competent patient regarding their goals for care, and review these regularly and as circumstances change. The advance directive or other records of the patient's wishes should be kept in the patient's file, with stickers indicating that the advance directive is available for ease of reference (paragraph 7.3.5). Reference is also made to the National Patients' Rights Charter ${ }^{[18]}$ introduced by the $\mathrm{NDoH}$ that requires patients to advise their healthcare provider of their wishes regarding death, and also that patients must care for medical records in their possession. ${ }^{[18]}$

Paragraph 7.3.7 suggests that advance directives should follow the guidelines of the General Medical Council ${ }^{[4]}$ in the UK, which state that an advance directive should include: (i) the patient's wishes and preferences regarding future treatment; (ii) beliefs or values that may influence the patient's decisions and preferences; (iii) family members or surrogates who should be involved in decision-making; (iv) any interventions that should be considered and implemented in case of emergency, such as cardiopulmonary resuscitation; $(v)$ the patient's preferred place of care; and (vi) the patient's need for religious, spiritual and other personal support.

Paragraph 7.3.8 states that, in a case of life-threatening illness towards the end of life, it is not uncommon for a patient's mental capacity to be impaired. When a patient becomes mentally incompetent, and an advance directive is available, healthcare practitioners providing palliative care should, in accordance with the WMA's Declaration of Venice, ${ }^{[16]}$ give effect to the patient's wishes provided that the directive is applicable to the present circumstances, and represents the patient's current wishes (paragraph 7.3.9). If no advance directive is available, and no surrogate has been appointed, the palliative care practitioner must, in consultation with the patient's family, determine the treatment option that is in the patient's best interests (paragraph 7.8.10).

In determining an incapacitated patient's best interests during the decision-making process, the palliative care practitioner must consider: (i) the medically appropriate treatment options available; (ii) the patient's previous requests when mentally competent; (iii) the practitioner's knowledge about the patient's background and preferences, in consultation with the patient's healthcare team; (iv) information regarding the patient's values, beliefs and preferences from third parties who may have other knowledge of the patient due to their relationship (e.g. a parent, sibling, child or spouse); and $(v)$ where more than one treatment option is reasonably in the patient's best interests, the practitioner must, in consultation with the patient or the patient's surrogate, choose the option that least restricts the patient's future choices (paragraph 7.3.11).

The palliative care practitioner must take appropriate action in the case of illegal, unethical or inappropriate behaviour that jeopardises the patient's best interests (paragraph 7.3.12).

\section{Communication}

Paragraph 7.4 relies on the HPCSA's 'Guidelines on withholding or withdrawing treatment.[19] Palliative care practitioners must communicate effectively and timeously with the patient and their family, and the rest of the palliative care team (paragraph 7.4.1). Palliative care providers must take into account the emotional toll that the circumstances regarding the use of palliative care may have on the patient and their family, and should communicate with them in an empathic and understandable manner (paragraph 7.4.2). If a patient's communication skills are compromised by their illness, palliative care practitioners must communicate with the patient through verbal and non-verbal means (paragraph 7.4.3). Irrespective of the patient's level of consciousness, palliative care practitioners should communicate proposed interventions to the patient, on the basis that hearing is thought to be the last sense to go in the dying process (paragraph 7.4.4).

\section{Beneficence and palliative care}

Paragraph 8.1.1 reminds practitioners that the principle of beneficence ${ }^{[11]}$ requires palliative care practitioners to do good for their patients. Palliative care practitioners must weigh up the costs, risks and benefits ${ }^{[4]}$ of palliative care options and recommend the most beneficial to the patient, while leaving the final decision to the patient or their surrogate (paragraph 8.1.2) From the time of diagnosis of a life-threatening condition, the palliative care practitioner must monitor the patient and keep abreast of relevant medical advancements, and ensure that their knowledge and understanding of palliative care is up to date (paragraph 8.1.3). ${ }^{[4]}$ Palliative care practitioners who cannot manage severe or refractory symptoms should refer the patient to a healthcare practitioner who is qualified to do so (paragraph 8.1.4). ${ }^{[4]}$

\section{Withholding or withdrawal of treatment}

Paragraph 8.2 refers palliative care practitioners to the HPCSA 'Guidelines on withholding or withdrawing treatment.'[19] The practitioner must balance the intended benefits of palliative care against the risks and burdens of treatment (paragraph 8.2.1). ${ }^{[4]}$ The quality of life which follows palliative treatment may sometimes raise questions about whether such treatment is in the patient's best interests (paragraph 8.2.2).

Treatment can legally and ethically be withheld or withdrawn if the patient refuses further treatment, or if further treatment is futile $\mathrm{e}^{[20]}$ or no longer in the patient's best interests, e.g. when treatment merely prolongs the dying process (paragraph 8.2.3). The decision to withhold or withdraw treatment must not be taken lightly, and must be considered by the palliative care practitioner in consultation with the patient and their family (paragraph 8.2.4). ${ }^{[19]}$ When the possibility of withholding or withdrawal of treatment arises, the practitioner should discuss with the patient and their family arrangements for basic care, and other appropriate treatments (paragraph 8.2.5). ${ }^{[19]}$

At the end of life, discussions about withholding or withdrawal of treatment should include plans to manage the final stages of the lives of patients, before they are incapacitated, and include personal matters such as wills and other concerns ${ }^{[19]}$ that patients believe are important, to ensure that they die with dignity (paragraph 8.2.6). ${ }^{[19]}$ When dealing with end-of-life decisions, palliative care practitioners must approach the withholding or withdrawal of treatment in a 
manner consistent with the HPSCA's 'Guidelines for the withholding and withdrawing of treatment (paragraph 8.2.7) ${ }^{\prime}{ }^{[19]}$

\section{Non-maleficence and palliative care}

Paragraph 9.1 refers to the HPSCA's 'Guidelines for the withholding and withdrawing of treatment, ${ }^{\prime[19]}$ and states that non-maleficence requires palliative care practitioners not to harm their patients, and complements beneficence and the balancing of risks and benefits. Euthanasia and physician-assisted suicide are often perceived as inconsistent with the principle of non-maleficence (paragraph 9.2). Both are legally prohibited in $\mathrm{SA}_{1}{ }^{[21]}$ and the courts frequently do not distinguish between the two when it comes to culpability (paragraph 9.3). Euthanasia is the employment of any medical intervention primarily aimed at ending a patient's life (e.g. giving a patient lethal drug or injection) (paragraph 9.4). Physician-assisted suicide occurs when the healthcare practitioner provides the means necessary to enable the patient to end their own life (e.g. handing a patient a lethal drug or prescribing a lethal drug for a patient) (paragraph 9.5). ${ }^{[21]}$

SA courts have held that active euthanasia and physician-assisted suicide are fundamentally incompatible with a practitioner's role as a healer, ${ }^{[21]}$ and a practitioner guilty of either is regarded as having acted unethically ${ }^{[16]}$ and unlawfully ${ }^{[2]}$ (paragraph 9.6). Due to the stress of extreme pain and the prospect of facing a life-threatening illness, a patient might request a palliative care practitioner to end their life (paragraph 9.7). The role of the practitioner in this instance is to discuss the concerns and fears that have led to the patient's request, and to provide alternative approaches to address these issues (paragraph 9.8).

When medical treatment relieves suffering but has the effect of accelerating the dying process, the palliative care practitioner must consider whether the palliative benefits justify the shortened life expectancy before pursuing such treatment (paragraph 9.9). Palliative care treatment where life is shortened as a side-effect is not regarded as unlawful or unethical ${ }^{[22]}$ - provided the patient or their surrogate has given informed consent (paragraph 9.10).

\section{Justice and fairness and palliative care}

Paragraph 10.1 records that 'palliative care needs to be provided in accordance with the principles of universal health coverage. ${ }^{[10]}$ This means that everyone, 'irrespective of income, disease type or age, should have access to a nationally determined set of basic health services, including palliative care' (paragraph 10.2). ${ }^{[10]}$ This is consistent with the SA Constitution. ${ }^{[13]}$ The WHO also states (paragraph 10.3) that 'financial and social protection systems need to take into account the human right to palliative care for poor and marginalised population groups.[10] Palliative care should be available to all patients from birth until death, and should be accessible at all levels of the healthcare service, and this is what the NDoH 's National Policy Framework and Strategy on Palliative Care ${ }^{[1]}$ aspires to do (paragraph 10.4).

Palliative care practitioners must treat patients equally and without discrimination (10.5). ${ }^{[10]}$ According to the NDoH 's National Policy Framework and Strategy on Palliative Care, ${ }^{[1]}$ palliative care practitioners must give special consideration to the following groups of people in providing palliative care services: (i) persons with disabilities; (ii) children (including neonates and adolescents); (iii) older persons, including those living in residential care settings and frail-care facilities; (iv) asylum seekers and refugees; ( $v$ ) inmates of correctional services; and (vi) persons in long-term care facilities such as TB and psychiatric hospitals or residential care facilities (paragraph 10.6)

Paragraph 10.7 concludes by reminding palliative care practitioners that they should use the country's limited healthcare resources responsibly, fairly and effectively to ensure that all patients receive appropriate palliative care.

Acknowledgements. Prof. Ames Dhai of the Steve Biko Centre for Bioethics, University of the Witwatersrand, Johannesburg, South Africa.

Author contributions. Equal contributions.

Funding. National Research Foundation.

Conflicts of interest. None.

1. National Department of Health. National Policy Framework and Strategy on Palliative Care 2017 - 2022. Pretoria: NDoH, 2017 https://hpca.co.za/download/ national-policy-framework-and-strategy-on-palliative-care-2017-2022/ (accessed 3 October 2018).

2. National Health Service Scotland. Scottish Palliative Care Guidelines. Edinburgh: NHS Scotland, 2014. http://www.palliativecareguidelines.scot.nhs.uk/guidelines/ about-the-guidelines.aspx (accessed 3 October 2018).

3. American Medical Association. Opinions on caring for patients at end of life. New York: AMA, 2016. https://www.ama-assn.org/sites/default/files/media-browser/ code-of-medical-ethics-chapter-5.pdf (accessed 3 October 2018).

4. General Medical Council. Treatment and care towards end of life: Good practice in decision-making. London: GMC, 2010. https://www.gmc-uk.org/ethicalguidance/ethical-guidance-for-doctors/treatment-and-care-towards-the-endof-life (accessed 3 October 2018).

5. National Clinical Programme for Palliative Care. Palliative care needs-assessment guidance. Dublin: NCPPC, 2014. https://www.hse.ie/eng/services/publications/ clinical-strategy-and-programmes/palliative-care-needs-assessment-guidance. pdf (accessed 3 October 2018).

6. Republic of Kenya Ministry of Health. National Palliative Care Guidelines. Nairobi: $\mathrm{MoH}$, 2013. http://kehpca.org/wp-content/uploads/National-Palliative-CareGuidelines-02.10.pdf (accessed 3 October 2018).

7. Australian Medical Association. Position statement on end-of-life care and advance care planning. Canberra: AMA, 2014. https://ama.com.au/position-statement/endlife-care-and-advance-care-planning-2014 (accessed 3 October 2018).

8. Canadian Medical Association. Palliative Care National Call to Action. Ottawa: CMA, 2015. https://www.cma.ca/Assets/assets-library/document/en/advocacy/ palliative-care-report-online-e.pdf (accessed 3 October 2018).

9. World Health Organization. Palliative care. http://www.who.int/cancer/palliative/ definition/en/ (accessed on 3 October 2018).

10. World Health Organization. Palliative care fact sheet. http://www.who.int/newsroom/fact-sheets/detail/palliative-care (accessed 3 October 2018).

11. Beauchamp TL, Childress JF. Principles of Biomedical Ethics, 5th edition. New York: Oxford University Press, 2001.

12. United Nations. International Covenant of Economic, Social and Cultural Rights. Geneva: UN, 1966. https://www.ohchr.org/en/professionalinterest/pages/cescr. aspx (accessed 4 October 2018).

13. South Africa. Constitution of the Republic of South Africa, 1996. https://www.gov. za/documents/constitution-republic-south-africa-1996 (accessed 3 October 2018).

14. Templeman J. An ethnographic study of critical care nurses' experience following the decision to withdraw life-sustaining treatment from patients in a UK intensive care unit. PhD thesis. Manchester: University of Salford, 2015. https://www.usir. salford.ac.uk/36188 (accessed 5 October 2018).

15. South Africa. National Health Act No. 61 of 2003. https://www.gov.za/documents/ national-health-act (accessed on 3 October 2018).

16. World Medical Association. Declaration of Venice on Terminal Illness. 1983 (revised 2006). https://www.wma.net/policies-post/wma-declaration-of-veniceon-terminal-illness/ (accessed 8 October 2018).

17. Health Professions Council of South Africa. Booklet 9: Seeking patients' informed consent: The ethical considerations. Pretoria: HPCSA, 2008. http://www.hpcsa. co.za/downloads/conduct_ethics/rules/generic_ethical_rules/booklet_9_ informed_consent.pdf (accessed 3 October 2018).

18. Health Professions Council of South Africa. Booklet 3: National Patients' Rights Charter. Pretoria: HPCSA, 2008. http://www.hpcsa.co.za/downloads/conduct_ ethics/rules/generic_ethical_rules/booklet_3_patients_rights_charter.pdf (accessed 3 October 2018).

19. Health Professions Council of South Africa. Booklet 12: Guidelines on withholding and withdrawing treatment. Pretoria: HPCSA, 2008. http://www.hpcsa.co.za/ Uploads/editor/UserFiles/downloads/conduct_ethics/Booklet 7.pdf (accessed 3 October 2018). 
20. Airedale NHSTrust v Bland 19931 All ER 821 (HL). https://www.globalhealthrights. org/wp-content/uploads/2013/01/HL-1993-Airedale-NHS-Trust-v.-Bland.pdf (accessed 3 October 2018).

21. Minister of Justice and Correctional Services and Others v Estate Late StranshamFord and Others [2016] ZASCA 197 (SCA). http://www.justice.gov.za/sca/ judgments/sca_2016/sca2016-197.pdf (accessed 8 October 2018).
22. McQuoid-Mason DJ. The Medical Profession and Medical Practice. Law of South Africa, 2nd edition. Vol 17 Part 2. Durban: LexisNexis, 2008: paragraph 54.

Accepted 27 February 2019. 